\title{
Common coding by pigeons in a many-to-one delayed matching task as evidenced by facilitation and interference effects
}

\author{
THOMAS R. ZENTALL and LOU M. SHERBURNE \\ University of Kentucky, Lexington, Kentucky \\ and \\ PETER J. URCUIOLI \\ Purdue University, West Lafayette, Indiana
}

\begin{abstract}
Pigeons were first trained on many-to-one delayed matching in which pairs of hue and lineorientation samples were associated with individual comparison stimuli. They were then trained to match two of the original samples (either hues or line orientations) to new comparisons, after which 2-sec delays were inserted between the samples and comparisons. In testing, the remaining samples were presented as interpolated stimuli during the delays. When the interpolated stimulus had been associated with the same comparison as the sample in many-to-one matching, performance was significantly more accurate than when it had been associated with a different comparison. This finding adds to the evidence that samples sharing common comparison associations are commonly coded.
\end{abstract}

With many-to-one (MTO) mapping, when two stimuli, $A$ and $B$, are independently associated with a common event, $C$, it is possible to show that an association has developed between $A$ and $B$-though $A$ and $B$ never have been presented together (Urcuioli, Zentall, Jackson-Smith, \& Steirn, 1989). Suggestive evidence for this phenomenon, called common coding, can be found in retention data from experiments involving delayed matching-to-sample (DMTS) in which there is an MTO mapping of samples onto comparisons. For example, differences between the slopes of retention functions that normally appear when sample discriminability is manipulated (hue samples vs. line-orientation samples) all but disappear in MTO DMTS (Zentall, Urcuioli, Jagielo, \& Jackson-Smith, 1989; see also Grant, 1982).

The similarity of retention functions that are typically quite different is consistent with the notion that samples associated with the same comparison are commonly coded. In other words, if memory for these samples consists of a common representation, one would expect that the rate of memory loss for the samples would be comparable, independently of the particular sample that gave rise to that representation.

Another source of evidence for common coding comes from reversal designs in which, following training on

This research was supported by National Science Foundation Grants BNS-8418275 and BNS-9019080 and National Institute of Mental Health Grant 45979 to T.R.Z. and P.J.U. The authors thank Karen L. Roper for her assistance. Correspondence should be addressed to T. R. Zentall, Department of Psychology, University of Kentucky, Lexington, KY 40506.
MTO DMTS, either all of the sample-comparison associations are reversed or only half are (Zentall, Steirn, Sherburne, \& Urcuioli, 1991, Experiment 1). The design of this experiment is presented in Table 1.

Under appropriate conditions, pigeons take longer to learn the partial reversal than the total reversal. Apparently, pigeons in the total reversal group maintain the common codes developed during original training and simply learn to reverse the responses to them. For pigeons in the partial reversal groups, on the other hand, membership in the commonly coded pairs has to be realigned.

Similar results, in which total reversals were learned faster than partial reversals, have been found with a simpler procedure in which, following training on two simple successive discriminations (e.g., red + , green - , and vertical + , horizontal - ), the reinforcement contingencies following either one or both discriminations were reversed (Zentall et al., 1991, Experiment 2).

Perhaps the strongest evidence for common coding comes from experiments involving transfer of training. For example, Urcuioli et al. (1989; cf. Wasserman, DeVolder, \& Coppage, 1992) trained pigeons to match red and vertical samples to a vertical comparison, and to match green and horizontal samples to a horizontal comparison. They then trained the pigeons to match one pair of the original samples (red and green) to new comparisons (circle and dot). When the pigeons were then tested with the other pair of samples (vertical and horizontal), they showed positive transfer if the new sample-comparison relations were consistent with the common codes and negative transfer if they were inconsistent with those codes. The design of this experiment is presented in Table 2. 
Table 1

Design of Common Coding Experiment Involving

Partial Versus Total Reversal Following Many-to-One Delayed Matching Training

\begin{tabular}{cccc}
\hline \multirow{2}{*}{ Phase 1 } & \multicolumn{3}{c}{ Phase 2 } \\
\cline { 2 - 4 } All Groups & Group Hue & Group Line & Group Hue-Line \\
\hline $\mathbf{R} \rightarrow \mathrm{C}$ & $\mathrm{R} \rightarrow \mathrm{D}$ & $\mathrm{R} \rightarrow \mathrm{C}$ & $\mathbf{R} \rightarrow \mathrm{D}$ \\
$\mathrm{G} \rightarrow \mathrm{D}$ & $\mathrm{G} \rightarrow \mathrm{C}$ & $\mathrm{G} \rightarrow \mathrm{D}$ & $\mathrm{G} \rightarrow \mathrm{C}$ \\
$\mathrm{V} \rightarrow \mathrm{C}$ & $\mathrm{V} \rightarrow \mathrm{C}$ & $\mathrm{V} \rightarrow \mathrm{D}$ & $\mathrm{V} \rightarrow \mathrm{D}$ \\
$\mathrm{H} \rightarrow \mathrm{D}$ & $\mathrm{H} \rightarrow \mathrm{D}$ & $\mathrm{H} \rightarrow \mathrm{C}$ & $\mathrm{H} \rightarrow \mathrm{C}$ \\
\hline
\end{tabular}

Note-The first letter of each pair represents the sample (R, red; G, green; V, vertical; $H$, horizontal), and the second letter represents the correct comparison (C, circle; D, dot).

Table 2

Design of Common Coding Experiment Involving Transfer of Training Following Many-to-One Delayed Matching Training

\begin{tabular}{cccc}
\multicolumn{1}{c}{ Group } & Phase 1 & Phase 2 & $\begin{array}{c}\text { Phase 3 } \\
\text { (Test) }\end{array}$ \\
\hline Consistent & $\mathrm{R} \rightarrow \mathrm{V}$ & & \\
& $\mathrm{G} \rightarrow \mathrm{H}$ & $\mathrm{R} \rightarrow \mathrm{C}$ & $\mathrm{V} \rightarrow \mathrm{C}$ \\
& $\mathrm{V} \rightarrow \mathrm{V}$ & $\mathrm{G} \rightarrow \mathrm{D}$ & $\mathrm{H} \rightarrow \mathrm{D}$ \\
& $\mathrm{H} \rightarrow \mathrm{H}$ & & \\
Inconsistent & $\mathrm{R} \rightarrow \mathrm{V}$ & & \\
& $\mathrm{G} \rightarrow \mathrm{H}$ & $\mathrm{R} \rightarrow \mathrm{D}$ & $\mathrm{V} \rightarrow \mathrm{C}$ \\
& $\mathrm{V} \rightarrow \mathrm{V}$ & $\mathrm{G} \rightarrow \mathrm{C}$ & $\mathrm{H} \rightarrow \mathrm{D}$ \\
& $\mathrm{H} \rightarrow \mathrm{H}$ & & \\
\hline
\end{tabular}

Note-The first letter of each pair represents the sample ( $R$, red; $G$, green; V, vertical; $H$, horizontal), and the second letter represents the correct comparison $(C$, circle; $D$, dot).

An alternative approach to the study of common coding is suggested by studies of retroactive inhibition in pigeons. In DMTS, the effect on pigeons' performance of presenting a stimulus during the retention interval depends on the similarity of the interpolated stimulus to the set of possible samples (Zentall, 1973). If MTO training leads to the formation of two stimulus classes through common coding, it follows that the effect of inserting a stimulus into the delay of a DMTS trial should vary, depending on whether that stimulus belongs to the same stimulus class as the sample or to a different class.

In the present experiment, pigeons initially learned MTO DMTS in which, for example, red and vertical samples were associated with a vertical comparison, and green and horizontal samples were associated with a horizontal comparison. They then learned to match one member of each sample pair to a new comparison, and delays were then inserted between the samples and comparisons of this interim task, in preparation for testing. In testing, the remaining member of each of the two stimulus pairs was presented during the delay. If this interpolated stimulus generated the same (common) code as did the sample itself, performance should be more accurate than if the interpolated stimulus generated the code associated with the other sample pair. In other words, the usual interference effect produced by stimuli interpolated during the delay in DMTS (Zentall, 1973) should also occur when stimuli commonly coded with the alternate sample are presented.
On the other hand, such interference effects should be diminished or even reversed by presentation of a stimulus commonly coded with the sample.

In some of the earlier common coding research, trained associations in original MTO DMTS training sometimes included identity matching trials (Urcuioli et al., 1989; Zentall et al., 1989). To assess the possible role played by identity relations in MTO common coding, half of the pigeons in the present experiment experienced lineorientation samples and correct comparisons that matched, whereas for the remaining pigeons, the line-orientation samples and correct comparisons did not match.

\section{METHOD}

\section{Subjects}

The subjects were 8 experimentally naive White Carneau pigeons purchased as retired breeders (over 5 years old) from the Palmetto Pigeon Plant (Sumter, SC). The pigeons were housed in individual wire cages with continuous access to water and grit. They were maintained throughout the experiment at $75 \%-80 \%$ of their freefeeding body weights. The colony room in which they were kept was maintained on a $12: 12$-h light:dark cycle.

\section{Apparatus}

The experiment was conducted in a sound-attenuated test chamber with a three-key response panel. The pigeons' compartment measured $33 \mathrm{~cm}$ high $\times 31 \mathrm{~cm}$ wide $\times 35 \mathrm{~cm}$ across the response panel. Three rectangular pecking keys $(3.0 \mathrm{~cm}$ wide $\times 2.5 \mathrm{~cm}$ high) were mounted side by side $(.5 \mathrm{~cm}$ apart) on the panel, with their bottom edges $21.0 \mathrm{~cm}$ from the wire-mesh floor. Behind each pecking key was a 12-stimulus in-line projector (Industrial Electronics Engineering, Series 10, with General Electric No. 1820 lamps) that projected red $(R)$ or green $(G)$ hues (Kodak Wratten filters Nos. 26 and 60 , respectively) and three white vertical (V) or horizontal $(H)$ lines (each $13 \mathrm{~mm}$ long $\times 3 \mathrm{~mm}$ wide and separated by $3 \mathrm{~mm}$ ) on the center key, and the same $\mathrm{V}$ or $\mathrm{H}$ lines or a white line-drawn circle (C, 16-mm outside diameter, 13- $\mathrm{mm}$ inside diameter) or a white dot (D, 5-mm diameter) on a black background, on the side keys. Access to a rear-mounted grain feeder filled with Purina Pro Grains was possible through a horizontally centered opening on the pecking panel $(6.0 \times 5.0 \mathrm{~cm})$ located midway between the bottom of the pecking keys and the floor. A feeder lamp was illuminated whenever the grain feeder was operated.

A shielded house light located $5.0 \mathrm{~cm}$ above the top edge of the center key provided general chamber illumination. White noise, at $72 \mathrm{~dB}$, and an exhaust fan provided masking noise. The experiment was controlled by a microcomputer located in an adjacent room.

\section{Procedure}

Pretraining. All pigeons were trained to eat from the feeder and were then shaped by the method of successive approximations to peck either $R$ ( 4 pigeons) or $G$ ( 4 pigeons) on the center key. Each peck resulted in a 2 -sec access to the reinforcer, followed by a 3$\mathrm{sec}$ intertrial interval (ITI) and a change in stimulus. Over the course of four 24-trial sessions, the number of pecks required for reinforcement was increased gradually to 10 . During each of these sessions, four stimuli ( $R, G, V$, and $H$ ) were each presented six times. On the following session, $\mathrm{V}$ and $\mathrm{H}$ stimuli were presented, 24 times each, on the left or the right side key, and a single peck to the lighted key was reinforced.

MTO DMTS training. On the day following pretraining, each pigeon began MTO DMTS training with 0-sec delays. Each trial began with presentation of $\mathrm{R}, \mathrm{G}, \mathrm{V}$, or $\mathrm{H}$ on the center key. Ten 
Table 3

Design of Present Common Coding Experiment

\begin{tabular}{|c|c|c|c|c|c|c|}
\hline \multirow{2}{*}{$\frac{\text { Group }}{\text { Match-hue }}$} & \multirow{2}{*}{$\frac{\text { Phase } 1}{R \rightarrow V}$} & \multicolumn{2}{|c|}{ Phase 2} & \multicolumn{2}{|c|}{ Phase 3 (test) } & \multirow{2}{*}{$\frac{\text { Effect }}{F}$} \\
\hline & & & & $\mathrm{R}-\mathrm{V} \rightarrow \mathrm{C}$ & $\mathrm{G}-\mathrm{H} \rightarrow \mathrm{D}$ & \\
\hline & $\mathbf{G} \rightarrow \mathbf{H}$ & $\mathbf{R}$ & $\rightarrow \mathrm{C}$ & $\mathbf{R}-\mathbf{H} \rightarrow \mathrm{C}$ & $G-V \rightarrow D$ & 1 \\
\hline & $\begin{array}{l}\mathrm{V} \rightarrow \mathrm{V} \\
\mathrm{H} \rightarrow \mathrm{H}\end{array}$ & $\mathbf{G}$ & $\rightarrow \mathrm{D}$ & $\mathrm{R} \rightarrow \mathrm{C}$ & $\mathrm{G} \rightarrow \mathrm{D}$ & B \\
\hline \multirow[t]{3}{*}{ Match-line } & $\mathrm{R} \rightarrow \mathrm{V}$ & & & $\mathrm{V}-\mathrm{R} \rightarrow \mathrm{C}$ & $\mathbf{H}-\mathbf{G} \rightarrow \mathrm{D}$ & $\mathbf{F}$ \\
\hline & $\mathrm{G} \rightarrow \mathrm{H}$ & V & $\rightarrow \mathrm{C}$ & $V-G \rightarrow C$ & $\mathbf{H}-\mathbf{R} \rightarrow \mathbf{D}$ & I \\
\hline & $\begin{array}{l}V \rightarrow V \\
H \rightarrow H\end{array}$ & $\mathrm{H}$ & $\rightarrow \mathrm{D}$ & $\mathrm{V} \rightarrow \mathrm{C}$ & $\mathrm{H} \rightarrow \mathrm{D}$ & B \\
\hline \multirow[t]{4}{*}{ Mismatch-hue } & $\mathrm{R} \rightarrow \mathrm{H}$ & & & $\mathrm{R}-\mathrm{V} \rightarrow \mathrm{C}$ & $\mathrm{G}-\mathrm{H} \rightarrow \mathrm{D}$ & $\mathrm{F}$ \\
\hline & $\mathrm{G} \rightarrow \mathrm{V}$ & $\mathbf{R}$ & $\rightarrow \mathrm{C}$ & $\mathrm{R}-\mathrm{H} \rightarrow \mathrm{C}$ & $G-V \rightarrow D$ & I \\
\hline & $\mathrm{V} \rightarrow \mathrm{H}$ & G & $\rightarrow \mathrm{D}$ & $\mathrm{R} \rightarrow \mathrm{C}$ & $\mathrm{G} \quad \rightarrow \mathrm{D}$ & B \\
\hline & $\mathbf{H} \rightarrow \mathrm{V}$ & & & & & \\
\hline \multirow[t]{3}{*}{ Mismatch-line } & $\mathbf{R} \rightarrow \mathbf{H}$ & & & $\mathrm{V}-\mathrm{R} \rightarrow \mathrm{C}$ & $\mathrm{H}-\mathrm{G} \rightarrow \mathrm{D}$ & $\mathbf{F}$ \\
\hline & $G \rightarrow V$ & V & $\rightarrow \mathrm{C}$ & $\mathrm{V}-\mathrm{G} \rightarrow \mathrm{C}$ & $\mathrm{H}-\mathrm{R} \rightarrow \mathrm{D}$ & I \\
\hline & $\mathrm{V} \rightarrow \mathrm{H}$ & $\mathbf{H}$ & $\rightarrow \mathrm{D}$ & $\mathrm{V} \rightarrow \mathrm{C}$ & $\mathbf{H} \rightarrow \mathrm{D}$ & B \\
\hline
\end{tabular}

Note-The first letter of each pair represents the sample (R, red; $G$, green; $\mathrm{V}$, vertical; $\mathrm{H}$, horizontal), the middle letter represents the stimulus interpolated during the delay, and the last letter represents the correct comparison ( $\mathrm{C}$, circle; $\mathrm{D}$, dot). The hypothesized common coding effect should either facilitate (F) or interfere with (I) performance, relative to baseline (B).

pecks to the sample resulted in sample offset and comparison ( $\mathrm{V}$ and $\mathbf{H}$ ) onset on the side keys. For half the pigeons, responding to the $\mathrm{V}$ comparison was reinforced following $\mathrm{R}$ and $\mathrm{V}$ samples, and responding to the $H$ comparison was reinforced following $G$ and $\mathrm{H}$ samples. For these pigeons, the line-orientation component of the MTO task involved a matching relation. For the remaining pigeons, responding to the $\mathrm{H}$ comparison was reinforced following $R$ and $V$ samples, and responding to the $V$ comparison was reinforced following $\mathrm{G}$ and $\mathrm{H}$ samples. For these pigeons, the lineorientation component of the MTO task involved a mismatching relation. The trial types presented during acquisition are presented in Table 3 (see the column labeled Phase 1). All comparison responses were followed by a 10 -sec ITI.

Sample stimulus and position of the correct comparison stimulus were counterbalanced across the 96 trials in each session. Trial types were randomly presented, with the constraint that on no more than three consecutive trials could a particular comparison or sidekey location be correct. Sessions were conducted 6 days a week. Each pigeon was trained on this 0 -sec-delay task to a performance criterion of $90 \%$ correct or better for two consecutive sessions, after which it was given an additional 20 sessions of training.

Phase 2 training. Upon completion of Phase 1, half the pigeons from each of the two training groups were trained on a DMTS task involving $R$ and $G$ samples and $C$ and $D$ comparisons, whereas the remaining pigeons had $\mathrm{V}$ and $\mathrm{H}$ samples and $\mathrm{C}$ and $\mathrm{D}$ comparisons. Each 96-trial session in Phase 2 was followed by 32 refresher trials on the original MTO DMTS task. Each pigeon was trained on its Phase 2 task with a 0 -sec delay until it matched at $90 \%$ correct or better accuracy for two consecutive sessions. It then received mixed-delay training involving twenty-four 0-sec-delay trials and seventy-two 2-sec-delay trials per session. Following each mixeddelay session, 32 refresher trials on the original MTO DMTS task were given. Mixed-delay training continued until performance on the 2 -sec-delay trials reached a criterion of $80 \%$ correct or better, for two consecutive sessions.

Testing. Following delay training, each pigeon received one 96trial test session. The samples and comparisons presented during testing were the same as those experienced during delay training. As during delay training, there were twenty-four 0 -sec and seventytwo 2-sec trials. On twenty-four 2-sec-delay trials, no stimulus was presented during the delay. The remaining 482 -sec-delay trials were test trials on which the sample stimuli from the original MTO DMTS task that were not used as samples in Phase 2 were each presented on the center key throughout the delay on 24 trials. The delay stimuli were counterbalanced over the various DMTS trial types such that the interpolated stimulus had been associated with the same Phase 1 comparison as the sample (consistent) on 24 trials and with the same Phase 1 comparison as the alternate sample (inconsistent) on the remaining 24 trials.

\section{RESULTS}

\section{MTO DMTS Training}

For all analyses, the .05 level of significance was adopted. In Phase 1, pigeons acquired hue-sample matching to a criterion of $90 \%$ correct faster than lineorientation matching ( 36.9 vs. 48.1 sessions), although a repeated measures analysis of variance (ANOVA) indicated that this difference did not reach statistical significance $[F(1,7)=2.82]$. (Two of the pigeons actually learned the line-orientation trial types faster than the hue trial types.) Similarly, although line-sample matching was learned somewhat slower than line-sample mismatching ( 52.5 vs. 43.8 sessions to criterion), this difference also was not statistically significant $(F<1)$.

\section{Phase 2 Training}

A two-way ANOVA performed on the Phase 2 acquisition data, with sample dimension (hues vs. lines) and Phase 1 line-sample task (matching vs. mismatching) as factors, indicated that the Phase 2 task was not learned significantly faster when the samples were hues than when they were lines $(F<1)$. The effect of line-sample matching/mismatching $(F<1)$ and the interaction $[F(1,4)=$ 2.11] were not significant, as well. A similar analysis performed on the delay-training data yielded similar results for the effects of having had matching during Phase 1 $(F<1)$ and of sample dimension $[F(1,4)=1.56]$, and for the interaction between these two factors $(F<1)$.

\section{Testing}

Overall, performance on consistent trials was more accurate than performance on inconsistent trials $(80.0 \%$ vs. $69.3 \%$ correct, respectively). Performance on control trials without an interpolated stimulus ( $78.1 \%$ correct) was comparable to performance on consistent trials. Post hoc contrasts across these means, using Rodger's (1975) procedure and tabled $F$ values, indicated that performance on consistent trials was not significantly different from that on control trials $(F<1)$, whereas performance on inconsistent trials was significantly less accurate than that on consistent and control trials combined $[F(1,7)=3.59]$. These statistical decisions imply that the ordering of matching accuracy during testing was as follows: inconsistent $<$ consistent $=$ control. Note that although presentation of unexpected stimuli during the delay might have been expected to have a general disruptive effect on performance, the analyses confirm that the disruption in performance was in fact specific only to the inconsistent trials. 


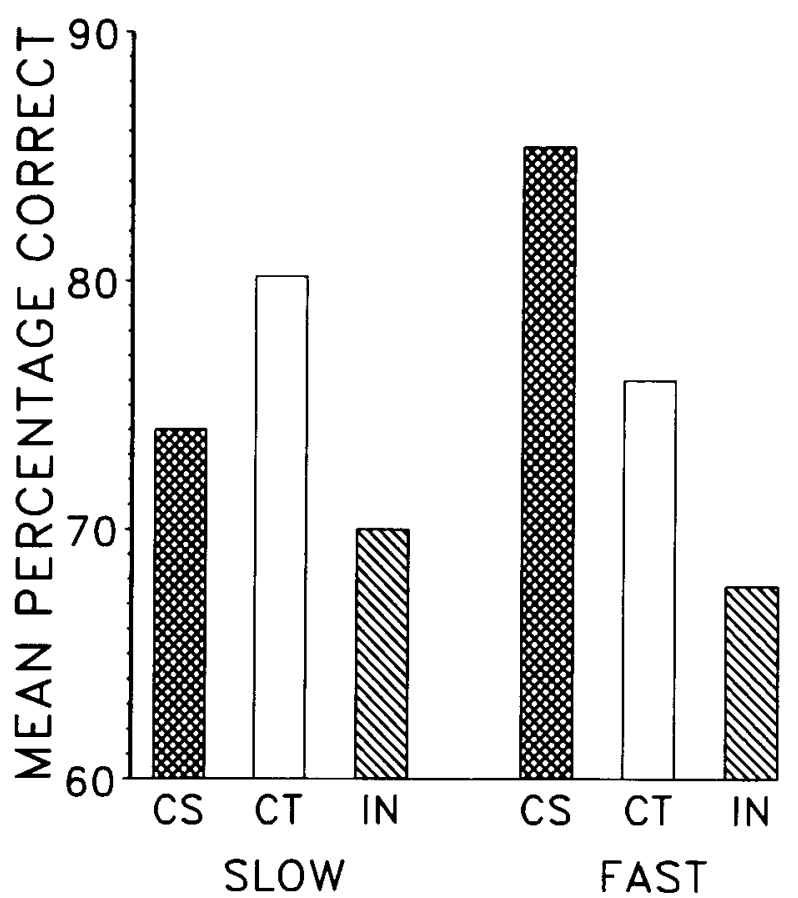

Figure 1. Test-trial performance on 2-sec-delay matching-to-sample trials for slow and fast learners with an interpolated stimulus hypothesized to have the same common code as that of the sample (consistent trials, CS), no interpolated stimulus (control trials, CT), and an interpolated stimulus hypothesized to have the same common code as that of the alternate sample (inconsistent trials, IN).

Comparison of the delay-training data with the transfer data indicated that pigeons that reached criterion on delay training in the fewest sessions were also the ones that showed the largest difference between consistent and inconsistent trial performance [Pearson's $r(7)=-.71$ ]. For this reason, the pigeons were divided into two groups (median split), according to their rates of delay acquisition. As can be seen in Figure 1, differences between performance on consistent and inconsistent trials were larger for fast learners (averaging 11.5 sessions to delayacquisition criterion) than for slow learners (averaging 29.5 sessions to delay-acquisition criterion). Furthermore, for slow learners, performance appeared to be disrupted on both consistent and inconsistent trials relative to control trials, whereas for fast learners, performance on control trials was midway between performance on consistent and inconsistent trials.

A two-way mixed ANOVA (with fast vs. slow delay learning, and consistent vs. inconsistent as factors) performed on these data indicated that performance on consistent trials was significantly better than performance on inconsistent trials $[F(1,6)=15.38]$. Furthermore, although performance on test trials by fast delay learners was not significantly better overall than that by slow learners, there was a significant delay-learning $\times$ consistentinconsistent interaction $[F(1,6)=7.54]$. For purposes of comparison, the data from control trials, in which no stim- ulus was presented during the delay, are also presented in Figure 1.

Two additional questions were asked. First, were differences in performance on consistent and inconsistent test trials affected by whether original training involved the matching as opposed to the mismatching of line orientations? A two-way mixed ANOVA indicated that the testtrial (consistent vs. inconsistent) $\times$ matching versus mismatching interaction was not significant $(F<1)$. Second, to what extent did the effect of interpolated stimuli depend on whether the samples were hues and the interpolated stimuli line orientations, or vice versa? Another two-way mixed ANOVA indicated that the test-trial $\times$ sample-dimension (hues vs. lines) interaction was not significant $(F<1)$. There was a significant effect of sample dimension, however $[F(1,6)=9.52]$. Performance by the pigeons that had hue-sample test trials $\mathbf{7 9 . 7 \%}$ correct) was significantly better than performance by those that had line-sample test trials $(69.3 \%$ correct $)$.

Finally, as mentioned before, 2 of the 8 pigeons learned the original line-orientation-sample trial types faster than they learned the hue-sample trial types. When the testtrial performances of these 2 birds were compared to those of the 6 birds that learned the hue-sample trial types first, a two-way ANOVA yielded a significant group $\times$ consistent-inconsistent interaction $[F(1,6)=17.29]$. Pigeons that acquired the hue-sample trial types first performed considerably better on consistent trials $(82.1 \%$ correct) than on inconsistent trials $(66.7 \%$ correct), whereas pigeons that acquired the line-orientation-sample trial types first showed little difference in performance on consistent and inconsistent trials $(72.9 \%$ and $77.1 \%$ correct, respectively).

\section{DISCUSSION}

The results of the present experiment support the view that training pigeons with MTO DMTS results in the common coding of samples associated with the same comparison. In the present experiment, one pair of the original samples was associated with a new pair of comparisons, and delays were inserted between the sample and comparison stimuli. When one of the remaining samples was then presented during the delay interval, performance was more accurate when that interpolated stimulus had shared a comparison association with the sample than when it had shared a comparison association with the alternate sample.

The present results also suggest that the rate at which pigeons acquire accurate delay performance may be a good predictor of transfer effects. Pigeons that acquired the 2-sec delay task quickly showed large effects of the interpolated stimulus, whereas those that acquired the delay task slowly did not. This relation between evidence of common coding on test trials and in delay-task acquisition is consistent with speculations about the nature of the common code (see Zentall, Sherburne, \& Urcuioli, 1993; Zentall et al., 1991). If the relatively difficult-to- 
remember lines (see Urcuioli \& Zentall, 1986; Zentall et al., 1989) were coded as the easier-to-remember hues, one would expect delay acquisition involving line samples to be facilitated. Furthermore, if the pigeons learned to code line samples as hues in original MTO training, one would not expect to see differences in the rate of delay-task acquisition as a function of the sample dimension. It is consistent with this prediction that, although the hue-sample component of the original MTO task was learned faster than the line-sample component, as expected, sample dimension did not play a role in the acquisition of the delay task.

Examination of Figure 1 also suggests that the interpolation of stimuli during the delay had a generally disruptive effect on DMTS performance of pigeons that learned the delay task slowly, but not of pigeons that learned the delay task quickly. These differential effects are consistent with the notion that the fast delay-task learners were commonly coding samples associated with the same comparison but the slow learners were not. For pigeons that commonly coded samples, the code associated with the sample on consistent test trials should have been the same as the code associated with the delay stimulus. On the other hand, for the slow learners (pigeons that, it has been suggested, did not commonly code the samples), the sample and the delay stimulus should have generated different codes on all test trials. When stimuli unrelated to an already-learned matching task are presented during the delay interval, matching performance tends to be disrupted (Zentall, 1973). Thus, test performance by the slow learners appears to be similar to what would be found with unrelated interpolated stimuli.

One might have expected that the interpolated stimulus presentations on inconsistent test trials would have had a more pronounced disruptive effect on matching performance than they did. For example, Grant (1984) reported that when two different sample hues were presented successively, pigeons tended to respond to comparisons on the basis of the second, rather than the first, sample. In the present experiment, however, the first sample was presented until the pigeon responded 10 times, whereas the second sample was presented for only $2 \mathrm{sec}$ (during which an average of 3.9 responses was made), and number of responses to the sample is known to affect matching accuracy (see Sacks, Kamil, \& Mack, 1972): Furthermore, the novelty of stimulus presentation during the delay, as well as any adjunctive delay behavior the pigeons might have developed during delay training (with unfilled delays), would have tended to reduce the magnitude of the interference effect on inconsistent test trials.

One of the purposes of the present experiment was to assess the role of identity matching during original MTO training. In earlier research involving the MTO DMTS design, in which an identity relation existed between one pair of samples and the correct comparison (see, e.g., Ur- cuioli et al., 1989; Zentall et al., 1989), one could argue that the relation between samples associated with the same comparison could have been established indirectly, through stimulus generalization. Given, for example, that responding to a vertical comparison is reinforced following a red or a vertical sample, the establishment of an association between the red sample and the vertical comparison may generalize to an association between the red sample and the vertical sample because of the similarity between the two vertical stimuli. The fact that line-orientation matching versus mismatching in Phase 1 had little effect on transfer performance suggests that the identity relation could not have mediated the common coding effect found in the present experiment. Furthermore, these findings suggest that sample-comparison identity relations probably did not play a role in earlier experiments in which evidence for the formation of stimulus classes has been found (e.g., Urcuioli et al., 1989; Zentall et al., 1989).

\section{REFERENCES}

Grant, D. S. (1982). Prospective versus retrospective coding of samples of stimuli, responses, and reinforcers in delayed matching. Learning \& Motivation, 13, 265-280.

GRANT, D. S. (1984). Directed forgetting and intertrial interference in pigeon delayed matching. Canadian Journal of Psychology, 38, 166-177.

RoDGER, R. S. (1975). The number of non-zero, post hoc contrasts from ANOVA and error rate: I. British Journal of Mathematical \& Statistical Psychology, 28, 71-78.

SACKs, R. A., KAMIL, A. C., \& MACK, R. (1972). The effects of fixedratio sample requirements on matching to sample in the pigeon. Psychonomic Science, 26, 291-293.

UrCuioli, P. J., \& Zentall, T. R. (1986). Retrospective memory in pigeons' delayed matching-to-sample. Journal of Experimental Psychology: Animal Behavior Processes, 12, 69-77.

Urcuiol, P. J., Zentall, T. R., Jackson-Smith, P., \& Steirn, J. N. (1989). Evidence for common coding in many-to-one matching: Retention, intertrial interference, and transfer. Joumal of Experimental Psychology: Animal Behavior Processes, 15, 264-273.

Wasserman, E. A., DeVolder, C. L., \& Coppage, D. J. (1992). Nonsimilarity-based conceptualization. Psychological Science, 3, 374-379.

ZENTALL, T. R. (1973). Memory in the pigeon: Retroactive inhibition in a delayed matching task. Bulletin of the Psychonomic Society, 1, 126-128.

Zentall, T. R., Sherburne, L. M., \& Urculoli, P. J. (1993). Common coding by pigeons of hedonic versus nonhedonic samples in a many-to-one delayed matching task. Manuscript submitted for publication.

Zentall, T. R., Steirn, J. N., Sherburne, L. M., \& UrCuiol, P. J. (1991). Common coding in pigeons assessed through partial versus total reversals of many-to-one conditional and simple discriminations. Journal of Experimental Psychology: Animal Behavior Processes, 17, 194-201.

Zentall, T. R., Urcuiou, P. J., Jagielo, J. A., \& Jackson-Smith, P. (1989). Interaction of sample dimension and sample-comparison mapping on pigeons' performance of delayed conditional discriminations. Animal Learning \& Behavior, 17, 172-178.

(Manuscript received October 14, 1992; revision accepted for publication February 1, 1993.) 